\title{
Temperature biofeedback and sleep: limited findings and methodological challenges
}

This article was published in the following Dove Press journal:

ChronoPhysiology and Therapy

9 October 2012

Number of times this article has been viewed

\author{
Geneviève Forest ${ }^{1,2}$ \\ Cameron van den $\mathrm{Heuvel}^{3}$ \\ Kurt Lushington ${ }^{4}$ \\ Joseph De Koninck ${ }^{2}$ \\ 'Sleep Laboratory, Département de \\ Psychoéducation et de Psychologie, \\ Université du Québec en Outaouais, \\ Gatineau, Québec, Canada; ${ }^{2}$ Sleep \\ and Dreams Laboratory, School of \\ Psychology, University of Ottawa, \\ Ottawa, Ontario, Canada; ${ }^{3}$ Research \\ Branch University of Adelaide, South \\ Australia, Australia; ${ }^{4}$ School of \\ Psychology, Social Work and Social \\ Policy, University of South Australia, \\ South Australia, Australia
}

\begin{abstract}
Given the close link between body temperature and sleep, the perspective of manipulating core and peripheral temperature by self-regulation techniques is very appealing. We report here on a series of attempts conducted independently in two laboratories to use self-regulation (biofeedback) of oral (central) and hand (peripheral) temperature, and measured the impact on sleep-onset latency, sleep architecture, and circadian phase. We found that hand temperature was more successful than oral temperature biofeedback. Moreover, an increase in hand temperature was associated with reduced sleep-onset latency. However, most participants found the procedure difficult to implement. The temperature response to biofeedback was reduced in the aged and weakest at the time of sleep onset, and there was not a systematic relationship between the change in temperature and change in sleep latency. Methodological limitations and individual differences may account for these results. Recommendations for future research are presented.
\end{abstract}

Keywords: biofeedback, core body temperature, sleep, circadian rhythm, sleep onset

\section{Introduction}

Sleep occurs at a critical point in the circadian temperature rhythm, ie, when the evening drop in the body temperature circadian curve appears. Moreover, sleep onset and slow-wave activity in sleep are strongly predicted by both the rate of nocturnal body temperature decline and the rate of distal heat loss at the feet, hand, and face. Therefore, there is a strong correlation between sleep architecture and circadian phase. However, the mechanisms underlying this association are not well documented and understood.

Recent findings have shown that thermoregulation plays an important role in sleep initiation and maintenance. Data from naturalistic studies have revealed that sleep onset is preceded by an increase in distal skin temperature and a decrease in core body temperature $(\mathrm{CBT}),{ }^{1-3}$ with the rate of CBT change strongly predicting latency to sleep onset. ${ }^{4-9}$ Correspondingly, raising distal skin temperature and lowering CBT using physical (eg, thermosuit, beverages, hot or warm baths/water, electric blankets) ${ }^{10-15}$ and pharmacologic agents (eg, melatonin, temazepam) has been shown to reduce sleep-onset latency. ${ }^{16-22}$ Taken together, these findings raise the possibility that thermoregulatory agents may be used to treat insomnia. A potential noninvasive self-regulating technique that could be successfully and easily used to manipulate distal skin temperature and CBT at bedtime is thermal biofeedback.

Voluntary control of skin temperature using biofeedback is well established and has been used successfully to treat a range of diseases (eg, migraine, phantom limb pain,
Correspondence: Geneviève Forest Département de Psychoéducation et de Psychologie, Université du Québec en Outaouais, Gatineau, Québec, Canada $\mathrm{Tel}+\mathrm{I} 8195953900$ extension 4434

Fax +I 8195952250

Email genevieve.forest@uqo.ca 
rheumatoid arthritis, hypertension, Reynaud's disease). ${ }^{23-29}$ By contrast, less is known about the effectiveness of thermal biofeedback as a treatment for insomnia. Two case studies report that hand temperature biofeedback was successful in normalizing sleep onset in a middle-aged patient with sleep-onset insomnia, ${ }^{25}$ and that foot warming improved sleep quality in a 21 -year-old good sleeper. ${ }^{30}$ These findings, although preliminary, indicate that thermal biofeedback may be an effective sleep-promoting agent. However, before investigating biofeedback in clinical groups, two issues need to be addressed. First, it remains to be established whether thermal biofeedback of peripheral temperature at bedtime can influence CBT under conditions that control for possible temperature confounds such as time-of-day effects and change in posture and activity. Second, it remains to be established whether self-modulation of distal skin temperature and CBT has an impact on sleep onset using objective sleep measures such as polysomnography (PSG).

We report here on attempts made independently in two laboratories to test whether the self-modulation of peripheral temperature using biofeedback has an impact on CBT and sleep onset in normal subjects. These studies were conducted at the Sleep and Dreams Laboratory of the University of Ottawa in Canada and at the Centre for Sleep Research of the University of South Australia, Adelaide. Some of those studies were the subject of presentations (abstracts) at international conferences in past years. ${ }^{31-35}$ Data accumulated in both sleep laboratories suggest that although thermal biofeedback has promise, two issues remain to be addressed in its development as a treatment for insomnia. First, methodological problems using thermal biofeedback around the time of sleep onset need to be addressed. Second, there is preliminary evidence that the temperature response to thermal biofeedback varies with circadian phase and is lowest at the time of sleep onset, thereby impacting treatment efficacy. Given the clinical implications and benefits of developing such a technique, the main objective of this paper is to present an integration of the findings of both laboratories in order to formulate recommendations for future studies.

\section{University of Ottawa studies Study I}

A healthy male (21 years old) was trained using visual imagery and autogenic biofeedback techniques via a computerized thermal biofeedback program, which provides visual feedback of hand temperature via a personal computer monitor (four temperature biofeedback systems, DOS version: Beta 1.7, Psytek Instrument Inc). Specifically, the subject was asked to produce any visual imagery associated with a warming of the hands (eg, hot water, sunbathing) or, on the contrary, cooling of the hands (eg, snow, cold water, ice). The subject could also use thoughts oriented on warming or cooling of the hands (eg, repeating to himself "my hands are cold, very cold; they are freezing"). The participant underwent three biofeedback training sessions per week for 4 weeks, which comprised 10 minutes of quiet rest (baseline measure), 20 minutes of biofeedback, and 10 minutes of quiet rest (return to baseline). He was instructed to raise and lower hand temperature for six trials each. To minimize the effect of posture on temperature ${ }^{37}$ and to better establish a baseline, the subject was seated in a comfortable reclining chair placed in front of the computer screen during biofeedback sessions and for the same duration (40 minutes) before the baseline night, during which no biofeedback took place.

Peripheral temperature was collected using glass tip temperature probes (sensitive to temperature changes of $0.055^{\circ} \mathrm{C}$ per second averaged over 60 seconds) attached to the palmar tip of each right and left index finger, to the left ankle (between the anklebone and the Achilles tendon), and under the tongue. Rectal temperature was continuously recorded using a rectal probe on a specially adapted Actillume by Ambulatory Monitoring (New York).

This study was conducted in a two-bedroom unit with an ambient temperature maintained at $23^{\circ} \mathrm{C} \pm 1^{\circ} \mathrm{C}$. The participant was instructed to maintain his regular sleep-wake pattern and to avoid naps for the duration of the experiment, which was monitored by a sleep diary. The participant underwent one adaptation/screening night followed by a baseline night in the laboratory (Eclipse ${ }^{\circledR}$ software v3.0 by Stellate System Inc, Montréal, Québec, Canada). This first night confirmed that no sleep disorder or circadian-related sleep problems were present. This was followed by biofeedback training and then two nonconsecutive experimental nights. On the experimental nights the participant employed biofeedback to raise hand temperature prior to bedtime, temperature was recorded continuously for 72 hours (24 hours prior to PSG, 24 hours PSG, and 24 hours home), and PSG sleep was collected using standard montage and scored according to standard criteria. ${ }^{38}$ The time taken from lights out to the onset of the first epoch of three continuous epochs of stage 1 sleep was used to define sleep onset.

The participant was able to increase his dominant hand temperature on the first experimental night (EN1) by $7^{\circ} \mathrm{C}\left(28.6^{\circ} \mathrm{C}-35.6^{\circ} \mathrm{C}\right)$ and on the second experimental night $(\mathrm{EN} 2)$ by $4.8^{\circ} \mathrm{C}\left(31.1^{\circ} \mathrm{C}-35.9^{\circ} \mathrm{C}\right)$. These distal skin temperature manipulations were accompanied by a decrease 
on EN1 in CBT of $0.4^{\circ} \mathrm{C}\left(37.1^{\circ} \mathrm{C}-36.7^{\circ} \mathrm{C}\right)$ and on EN2 of $0.3^{\circ} \mathrm{C}\left(37.2^{\circ} \mathrm{C}-36.9^{\circ} \mathrm{C}\right)$. Baseline night $(\mathrm{BN}) \mathrm{CBT}$ was a constant $37.5^{\circ} \mathrm{C}$ at the same hour (CBT changes over time are expressed in Figure 1). The temperature changes on both EN were accompanied by a decrease in sleep-onset latency $(\mathrm{BN}=19.5$ versus $\mathrm{EN} 1=14.5$ and $\mathrm{EN} 2=8$ minutes $)$.

\section{Study 2}

Ten participants were recruited (exclusion criteria were personal or familial history of psychiatric or sleep disorders, drug or alcohol abuse, any medication or oral contraceptive use). During the study, all subjects were asked to refrain from caffeine intake (food or beverage containing caffeine) except for one cup of coffee in the morning. A regular sleep-wake schedule was recommended for at least 2 weeks before the experiment. Compliance was verified using a sleep diary for 7 days prior to the study. Only two subjects (a 22-year-old female and a 20 -year-old male) were successful at reaching biofeedback criteria (ie, raising and lowering dominant hand temperature $\geq 1.5^{\circ} \mathrm{C}$ ). ${ }^{36}$ These two participants subsequently underwent a similar protocol to that of study 1 but with additional experimental nights, to examine the impact of lowering hand temperature at bedtime. The final 11-night protocol consisted of adaptation/screening (no sleep and/or circadian rhythm disorders were identified) and baseline PSG nights, followed a week later by baseline and two experimental and follow-up nights, which was then repeated a week later. The order of raising and lowering hand temperature was counterbalanced, and rectal temperature was collected during the study and in the week between experimental conditions.

The effects of hand temperature biofeedback on CBT and sleep onset for the two successful participants are provided in Table 1. Both participants successfully manipulated their hand temperature during training, but this did not generalize to bedtime on the experimental nights, and no systematic effect was observed on CBT and sleep onset. Paradoxically, and regardless of condition, on the experimental nights compared with baseline nights, the fall in CBT was greater and sleep-onset latency shorter.

\section{Study 3}

Based on the findings from study 2, it was hypothesized that oral temperature biofeedback may be a more effective strategy. Two young healthy male participants (a 21-year-old and a 22-year-old) underwent the 11-night sleep protocol described in study 2 , using the same exclusion criteria and experimental conditions. Participants had limited success either raising or lowering oral temperature on both the training and experimental nights with minimal effects on CBT and sleep latency (see Table 2).

\section{University of South Australia}

Using similar methodologies to those described by the Ottawa group, the University of South Australia (UniSA) "Adelaide" group have also conducted two studies using thermal biofeedback.

\section{Study I}

Twelve healthy participants underwent biofeedback training involving one training session in the laboratory followed by a 2 -week training protocol at home. The subjects were screened for mood disorders using the Beck Depression Inventory, the Stait Trait Anxiety Inventory, and the General Health Questionnaire and Illness Behaviour Questionnaire. Sleep was assessed using the Pittsburgh Sleep Quality Index and a 7-day sleep log. Subjects were furthered screened to exclude those with sleep disorders and circadian-related sleep problems. All subjects were medication free for 2 weeks prior to and during the studies, including hypnotics and contraceptives. Participants were required to meet

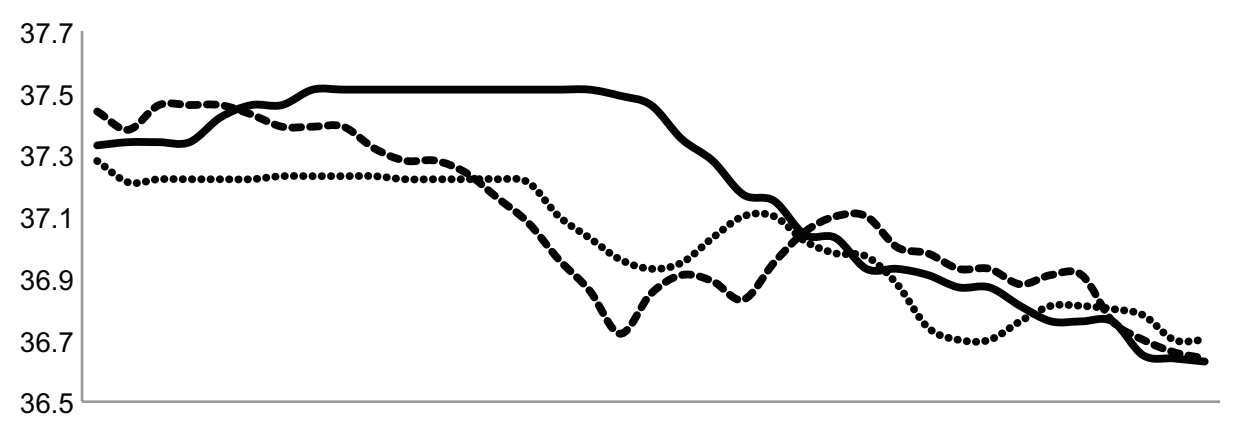

22h00 22h15 22h30 22h45 23h00 23h15 23h30 23h45 00h00 00h15 00h30 00h45 $01 \mathrm{~h} 00$

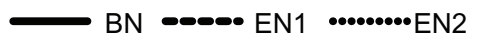

Figure I Core body temperature changes on baseline night (BN), on the first experimental night (ENI), and on the second experimental night (EN2). 
Table I Dominant hand temperature changes (in ${ }^{\circ} \mathrm{C}$ ) during biofeedback and the concomitant effect on CBT (rectal) and subsequent sleep onset latencies

\begin{tabular}{|c|c|c|c|c|c|c|}
\hline & \multicolumn{2}{|c|}{ Dominant hand temperature } & \multicolumn{2}{|c|}{ CBT (rectal) } & \multicolumn{2}{|c|}{ Sleep onset latencies } \\
\hline & Subject I & Subject 2 & Subject I & Subject 2 & Subject I & Subject 2 \\
\hline Baseline nights & NA & NA & -0.06 & -0.12 & $22.7 \mathrm{~min}$ & $3.5 \mathrm{~min}$ \\
\hline Experimental night $(\downarrow)$ & -0.73 & -1.13 & -0.18 & NA & $9.0 \mathrm{~min}$ & $5.0 \mathrm{~min}$ \\
\hline Experimental night $(\downarrow)$ & -2.6 & -3.29 & -0.29 & -0.12 & $4.3 \mathrm{~min}$ & $2.0 \mathrm{~min}$ \\
\hline Experimental night $(\uparrow)$ & -1.83 & 4.48 & -0.30 & -0.28 & $8.3 \mathrm{~min}$ & $1.0 \mathrm{~min}$ \\
\hline Experimental night $(\uparrow)$ & 3.05 & 3.43 & -0.30 & -0.34 & $6.3 \mathrm{~min}$ & $1.5 \mathrm{~min}$ \\
\hline
\end{tabular}

a $\pm 1.5^{\circ} \mathrm{C}$ change in hand temperature biofeedback criteria before proceeding to the experimental conditions. Three participants were unable to meet this criterion and were excluded, leaving nine participants (mean \pm standard deviation age $=26.6 \pm 6.1$ years; four female). A small portable temperature biofeedback instrument with a digital readout and a 3M-temperature probe was used for biofeedback training (Stress Thermometer Model SC911: Conscious Living Foundation, Drain, OR). Participants were instructed to lower hand temperature in the first week and raise hand temperature in the second week, and were encouraged to use whatever temperature-changing strategy was effective, including visual imagery and/or thoughts. To minimize the effect of posture on temperature and to better establish a baseline, participants were instructed to lie down for at least 15 minutes prior to biofeedback training.

The study was conducted in a four-bed sleep unit where ambient temperature was maintained at $23^{\circ} \mathrm{C} \pm 1^{\circ} \mathrm{C}$. The bedding consisted of a light sheet, and participant clothing was restricted to a T-shirt and shorts. Peripheral temperature was recorded using thermistor probes (Steri-Probe 499B, Cincinnati Sub-Zero Products, Cincinnati, $\mathrm{OH}$ ) attached to the palmar tip of the index finger of the dominant hand (right hand in all cases). Temperature was sampled at 1-second intervals. All female participants were tested within 14 days following menses. A modified multiple sleep latency test protocol with trials extended to 30 minutes was used to measure sleep onset. PSG data were acquired using a Compumedics
S-series system (Melbourne, Australia) and collected using a standard PSG montage. Sleep was scored using standard criteria, ${ }^{38}$ and the time taken from lights out to the onset of the first epoch of three continuous epochs of stage 1 sleep was used to define sleep onset. Participants were woken after three consecutive epochs of sleep to minimize the accumulation of sleep. Sleep onset was recorded as 30 minutes if participants did not fall asleep.

Participants underwent three experimental conditions scheduled at least 2 days apart, lowered, raised, and control temperature, which were counterbalanced using a Latinsquare design. Participants were instructed to maintain their regular sleep-wake pattern between conditions, and this was monitored using a sleep diary. Each experimental condition involved participants remaining supine in bed from 13:00 to 23:00. They participated in a habituation biofeedback session at 14:00 followed by four biofeedback sessions scheduled at 16:00, 18:00, 20:00, and 22:00. Each trial consisted of 10 minutes of quiet rest (baseline), 14 minutes of biofeedback, and a 30-minute sleep latency trial. Depending on the experimental condition, participants were instructed at the start of each trial to raise, lower, or not attempt to alter hand temperature. Participants were permitted to use the biofeedback devices during the biofeedback session but were instructed to detach the temperature probe at lights out for the multiple sleep latency tests. In between trials, participants were permitted to engage in quiet activities such as reading and watching television. Alertness was monitored using

Table 2 Oral temperature biofeedback changes (in ${ }^{\circ} \mathrm{C}$ ) during biofeedback and the concomitant effect on CBT (rectal) and subsequent sleep onset latencies

\begin{tabular}{|c|c|c|c|c|c|c|}
\hline & \multicolumn{2}{|c|}{ Oral temperature } & \multicolumn{2}{|c|}{ CBT (rectal) } & \multicolumn{2}{|c|}{ Sleep onset latencies } \\
\hline & Subject I & Subject 2 & Subject I & Subject 2 & Subject I & Subject 2 \\
\hline Baseline nights & NA & NA & -0.12 & -0.12 & $6.25 \mathrm{~min}$ & $15.0 \mathrm{~min}$ \\
\hline Experimental night $(\downarrow)$ & -0.25 & -0.22 & -0.06 & -0.12 & $6.25 \mathrm{~min}$ & $6.0 \mathrm{~min}$ \\
\hline Experimental night $(\downarrow)$ & NA & -0.02 & NA & -0.05 & NA & $21.0 \mathrm{~min}$ \\
\hline Experimental night $(\uparrow)$ & 0.01 & -0.26 & -0.12 & -0.17 & $5.0 \mathrm{~min}$ & $6.5 \mathrm{~min}$ \\
\hline Experimental night $(\uparrow)$ & 0.07 & NA & -0.12 & NA & $7.5 \mathrm{~min}$ & NA \\
\hline
\end{tabular}


PSG and activity using closed-circuit television. Participants received cold snacks at 17:00, 19:00, and 21:00.

To control for intercondition differences, the temperature data for each session were expressed relative to each individual's baseline average. The mean relative dominant hand temperature over the biofeedback period and the sleep-onset latencies were analyzed using two analyses of variance: time of day $(16: 00,18: 00,20: 00$, and 22:00) and condition (raising, lowering, and control). $T$-tests were performed in post-hoc analysis. The relationship between the changes in hand temperature following biofeedback and changes in sleep-onset latencies were examined using Pearson- $r$ correlations.

The detailed results of the hand temperature over the 14-minute biofeedback period for each time of day and each condition are illustrated in Figure 2 and presented in Table 3. Analyses revealed a significant main effect for condition and a significant condition by time-of-day interaction. Post-hoc comparison revealed that, compared with baseline, participants were more effective at raising than lowering hand temperature, and the magnitude of change was greater at 16:00 and 18:00 compared with at 20:00 and 22:00 $(P<0.05$ were appropriate). A significant main effect was observed for condition, and post-hoc tests revealed that sleep onset was shorter in the raising compared with control and lowering conditions ( $P<0.05$ were appropriate). Finally, and regardless of trial, no significant relationships were observed between the changes in hand temperature and sleep-onset latency (all $r<0.2$ ).

\section{Study 2}

Sixteen older participants (mean age $=65.6 \pm 8.7$ years; eleven females) were screened for the same exclusion criteria as described in study 1. Apart from three modifications, the protocol was the same as that described in study 1: (1) because the presence of the biofeedback device was thought to potentially disturb sleep, it was removed from the bedroom on experimental nights; (2) because participants were unable to meet the $\pm 1.5^{\circ} \mathrm{C}$ change in hand temperature biofeedback criteria, this was reduced to $\pm 0.5^{\circ} \mathrm{C}$; and (3) the experimental trial baseline period was extended from 10 to 15 minutes to better allow the aged participants to settle.

The mean (standard deviation and analysis of variance) results for study 2 are reported in Table 4. No significant main or interaction effects were observed. In addition, and regardless of trial, no significant relationship was observed between the pre-post change following biofeedback in hand temperature and subsequent sleep latency (all $r<0.2$ ).
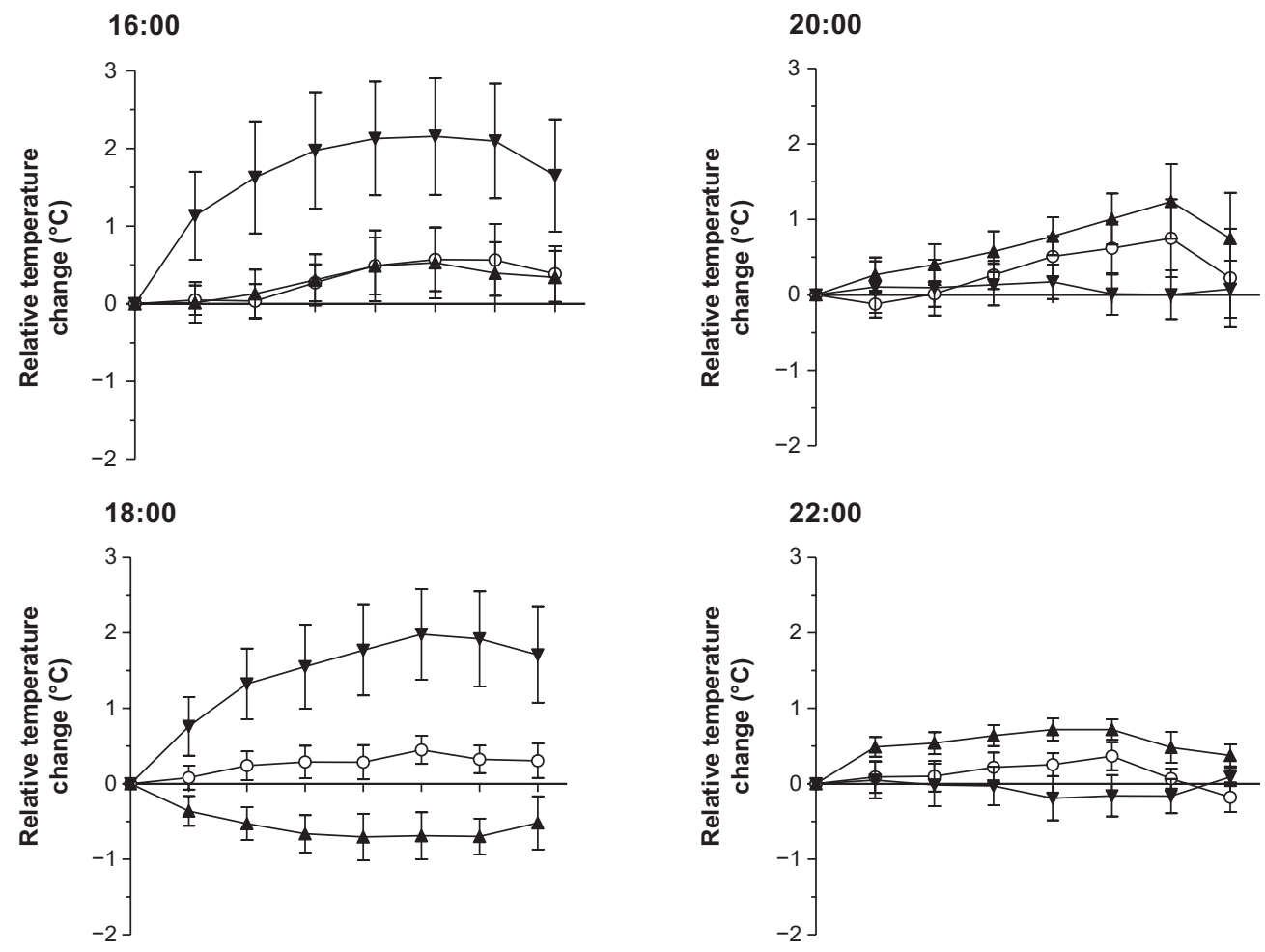

Figure 2 The relative (adjusted to baseline) dominant hand temperature curves over the biofeedback 0- to I4-minute period for each time of day: I6:00, I8:00, 20:00, and 22:00. Notes: $\Delta$ indicates raising; $\nabla$ indicates lowering dominant hand temperature; ${ }^{\circ}$ indicates control at $0,+2,+4,+6+8+10,+12$ and +14 min after commencing biofeedback. Data expressed as mean (SD) relative to baseline values. 
Table 3 In young adults

\begin{tabular}{|c|c|c|c|c|c|c|}
\hline \multirow{2}{*}{$\begin{array}{l}\text { Dependent } \\
\text { variable }\end{array}$} & \multicolumn{3}{|l|}{ Condition } & \multicolumn{3}{|c|}{ ANOVA F-Values } \\
\hline & Raising & Control & Lowering & Condition & Time-of-day & $\begin{array}{l}\text { Condition by } \\
\text { time-of-day }\end{array}$ \\
\hline \multicolumn{7}{|l|}{ Temp $\left({ }^{\circ} \mathrm{C}\right)$} \\
\hline $16: 00 \mathrm{~h}$ & $1.97(2.32)$ & $0.34(0.75)$ & $0.36(1.01)$ & $8.8^{* *}$ & 1.8 & $2.7^{*}$ \\
\hline $18: 00 \mathrm{~h}$ & $1.77(1.69)$ & $0.28(0.46)$ & $-0.70(0.73)$ & & & \\
\hline $20: 00 \mathrm{~h}$ & $0.67(1.02)$ & $0.32(0.88)$ & $0.05(0.90)$ & & & \\
\hline $22: 00 \mathrm{~h}$ & $0.57(0.40)$ & $0.13(0.51)$ & $-0.06(0.65)$ & & & \\
\hline Mean (SD) & $1.24(1.60)$ & $0.27(0.65)$ & $-0.09(0.89)$ & & & \\
\hline \multicolumn{7}{|l|}{ SO (min) } \\
\hline $16: 00 \mathrm{~h}$ & $9.4(9.0)$ & $21.8(8.9)$ & $21.5(10.7)$ & $6.5^{* *}$ & 1.9 & 2.2 \\
\hline $18: 00 \mathrm{~h}$ & $19.2(10.1)$ & $22.3(8.0)$ & $24.5(10.6)$ & & & \\
\hline $20: 00 \mathrm{~h}$ & $22.6(I 1.30)$ & $24.0(8.9)$ & $26.4(7.1)$ & & & \\
\hline $22: 00 \mathrm{~h}$ & $14.7(\mid I .9)$ & $21.6(12.6)$ & $27.1(6.8)$ & & & \\
\hline
\end{tabular}

Notes: Mean (SD) dominant hand temperature $\left({ }^{\circ} \mathrm{C}\right)$ over the biofeedback period relative to baseline values, and sleep onset (SO) latency for the raising, control and lowering biofeedback conditions at each time-of-day (16:00, 18:00, 20:00, and 22:00 h) and F-Values from ANOVA. Nb $* P<0.05$; $* * P<0.01$.

\section{Discussion}

The objective of this paper was to present and summarize the results of multiple experiments made in two separate sleep laboratories over a period of 20 years, examining thermal biofeedback as a method for shortening sleep latency and therefore as a possible treatment for insomnia. Although theoretically attractive, the results from both sleep laboratories have been disappointing. The majority of participants found thermal biofeedback difficult to implement, and the impact on sleep latency was unsystematic. However, despite these limitations there remains strong evidence that thermoregulation plays an important role in sleep initiation and maintenance, and, as such, thermal biofeedback remains a theoretically attractive area for future investigation. In the subsequent sections we discuss the methodological limitations experienced by our groups so that this may guide future research in this promising but unfilled area of investigation.

There were several findings of note. First, hand temperature biofeedback is more easily implemented than oral temperature biofeedback, and we encourage researchers to continue to explore the latter modality. Second, there was considerable interindividual variability in thermal biofeedback mastery. This variability appears unrelated to the number of training sessions. Four to six training sessions are typically reported to be sufficient, and adding more training sessions is not reported to increase the magnitude of temperature changes. ${ }^{36}$ Similarly, the duration of training sessions does not appear to be a major limitation, with the Ottawa group scheduling 20 minutes and the Adelaide group 14 minutes with comparable efficiency. Third, we did not look at the possible association between the relaxation

Table 4 In adults $>55 y$

\begin{tabular}{|c|c|c|c|c|c|c|}
\hline \multirow{2}{*}{$\begin{array}{l}\text { Dependent } \\
\text { variable }\end{array}$} & \multicolumn{3}{|l|}{ Condition } & \multicolumn{3}{|c|}{ ANOVA F-Values } \\
\hline & Raising & Control & Lowering & Condition & Time-of-day & $\begin{array}{l}\text { Conditionby } \\
\text { time-of-day }\end{array}$ \\
\hline \multicolumn{7}{|l|}{ Temp $\left({ }^{\circ} \mathrm{C}\right)$} \\
\hline $16: 00 \mathrm{~h}$ & $0.68(0.96)$ & $0.14(0.38)$ & $0.14(0.59)$ & 2.5 & $\mathrm{I} .4$ & 1.4 \\
\hline $18: 00 \mathrm{~h}$ & $0.66(0.66)$ & $0.05(1.00)$ & $0.23(0.73)$ & & & \\
\hline $20: 00 \mathrm{~h}$ & $0.60(0.93)$ & $0.64(0.73)$ & $0.46(1.10)$ & & & \\
\hline $22: 00 \mathrm{~h}$ & $0.64(0.63)$ & $0.58(0.82)$ & $0.34(0.62)$ & & & \\
\hline Mean (SD) & $0.64(0.79)$ & $0.35(0.79)$ & $0.29(0.77)$ & & & \\
\hline \multicolumn{7}{|l|}{ SO $(\min )$} \\
\hline $16: 00 \mathrm{~h}$ & $17.5(12.1)$ & I 4.7 (9.8) & I8.7 (II.8) & 0.1 & 0.1 & 0.6 \\
\hline $18: 00 \mathrm{~h}$ & $16.8(10.6)$ & $18.6(10.9)$ & I8.8 (9.5) & & & \\
\hline $20: 00 \mathrm{~h}$ & $18.6(10.4)$ & I8.I (9.8) & $17.0(9.6)$ & & & \\
\hline $22: 00 \mathrm{~h}$ & $16.3(11.3)$ & $17.9(8.1)$ & I8.I (10.0) & & & \\
\hline
\end{tabular}

Notes: mean (SD) dominant hand temperature (in ${ }^{\circ} \mathrm{C}$ ) over the biofeedback period relative to baseline values, and sleep onset (SO) latency for the raising, control and lowering biofeedback conditions at each time-of-day (16:00, 18:00, 20:00, and 22:00 h) and F-Values from ANOVA. 
effects of the sessions themselves and the efficiency of the temperature biofeedback and subsequent sleep onset. This should be more closely examined in further studies. Fourth, the response to thermal biofeedback may reduce with age. Despite better adherence to the training protocol, the older participants in the Adelaide studies found raising and, especially, lowering hand temperature more challenging than the younger participants, which may limit the utility of thermal self-regulation in the elderly. Fifth, it is also our experience that without a device present for self-monitoring, the capacity to generalize the daytime training to the presleep period may be limited. Sixth is the timing of biofeedback. Van Someren ${ }^{3}$ has argued that "... the timing of core-body temperature increasing methods is of crucial importance" and if too close to sleep onset the participant may feel too aroused to go to sleep and "... if the manipulation is timed too long before sleep onset, core temperature may already be back on the baseline level at the onset of sleep, and no increase heat loss during sleep will be attained." The timing of thermal biofeedback in both laboratories was just prior to lights out, and as a general observation participants did report increased relaxation. An allied issue is whether there is a time-of-day response to thermal biofeedback. At bedtime, distal skin temperature may naturally be approaching a maximal level, and therefore peripheral temperature modifications might be limited by a ceiling effect in the heat loss process. Results from the Adelaide group strongly suggest that there is a circadian influence on the ability to manipulate hand temperature, which is weakest at the time of sleep onset. Finally, it should be noted that all participants were healthy good sleepers. A recent study by van den Heuvel et $\mathrm{al}^{39}$ suggests that sleep-onset insomniacs may have an altered thermoregulatory process at bedtime. ${ }^{40}$ Given that the physiologic mechanisms of temperature biofeedback are different in normal compared with patients with thermoregulatory problems,${ }^{36}$ thermal biofeedback may have a different impact on insomniacs. This is worth pursuing.

\section{Conclusion}

The limited success observed in the present studies suggests that the physiologic processes involved in peripheral thermal biofeedback and its relationship with sleep are more complex than what was expected. Despite restricted methodological controls and binding protocols, many factors related to the biofeedback technique itself, individual differences, differences in circadian typology, physiologic mechanisms of vasodilatation, and timing of temperature manipulations prevent the usefulness of thermal biofeedback in shortening sleep onset. Given the potential benefits that may result from the development of an efficient self-regulation technique to improve sleep in specific patients, we think that these factors need to be addressed in futures studies.

\section{Acknowledgments}

The University of Ottawa work was supported by the Natural Sciences and Engineering Research Council of Canada. We would like to acknowledge the participation of the following students and colleagues: University of Ottawa: Michelle Castonguay, Yves Côté, Carole Couture-Côté, Daniel Forget, Anne-Karine Gauthier, Jean Grenier, Anik Gosselin, Gilles Hébert Lynne Lamarche, and, finally, Paul Swingle particularly for the development of the biofeedback technique used; University of South Australian: Joanna Bothma, Therese Faulkner, Saul Gilbert, Helen Greeneklee, Andrea Hubbard, Kimmy Luk, Wendy Maxwell, Srdjan Vajdic, and Melinda Veltmeyer.

\section{Disclosure}

The authors report no conflicts of interest in this work.

\section{References}

1. Lack L, Gradisar M. Acute finger temperature changes preceding sleep onsets over a 45-h period. J Sleep Res. 2002;11:275-282.

2. Murphy P, Campbell SS. Nighttime drop in body temperature: a physiological trigger for sleep onset? Sleep. 1997;20:505-511.

3. Van Someren EJW. More than a marker: interaction between the circadian regulation of temperature and sleep, age-related changes, and treatment possibilities. Chronobiol Int. 2000;17:313-354.

4. Gradisar M, Lack L. Relationship between the circadian rhythms of finger temperature, core temperature, sleep latency, and subjective sleepiness. J Biol Rhythms. 2004;19:157-163.

5. Käuchi K. The thermophysiological cascade leading to sleep initiation in relation to phase of entrainment. Sleep Med Rev. 2007;11:439-451.

6. Kräuchi K, Cajochen C, Werth E, Wirz-Justice A. Warm feet promote the rapid onset of sleep. Nature. 1999;401:36-37.

7. Kräuchi K, Cajochen C, Werth E, Wirz-Justice A. Functional link between distal vasodilatation and sleep-onset latency? Am J Physiol. 2000;278:R741-R748.

8. Kräuchi K, Wirz-Justice A. Circadian rhythm of heat production, heart rate, and skin and core temperature under unmasking conditions in men. Am J Physiol. 1994:267:R819-R829.

9. van den Heuvel CJ, Noone JT, Lushington K, Dawson D. Changes in sleepiness and body temperature precede nocturnal sleep onset: evidence from a polysomnographic study in young men. J Sleep Res. 1998;7:159-166.

10. Bunnell DE, Agnew JA, Horvath SM, Jopson L, Wills M. Passive body heating and sleep: influence of proximity to sleep. Sleep. 1988;11: 210-219.

11. Ebben MR, Spielman AJ. The effects of distal limb warming on sleep latency. Int J Behav Med. 2006;13:221-228.

12. Fletcher A, van den Heuvel C, Dawson D. Sleeping with an electric blanket: effects on core temperature, sleep, and melatonin in young adults. Sleep. 1999;22:313-318.

13. Raymann RJE, Swaab DF, Van Someren EJW. Cutanuous warming promotes sleep onset. Am J Physiol. 2005;288:R1589-R1597. 
14. Raymann RJE, Swaab DF, Van Someren EJW. Skin temperature and sleep-onset latency: changes with age and insomnia. Physiol Behav. 2007;90:257-266.

15. Raymann RJE, Swaab DF, Van Someren EJW. Skin deep: enhanced sleep depth by cutaneous temperature manipulation. Brain. 2008;131: 500-513.

16. Brzezinski A, Vangel MG, Wurtman RJ, et al. Effects of exogenous melatonin on sleep: a meta-analysis. Sleep Med Rev. 2005;9:41-50.

17. Gilbert SS, van den Heuvel CJ, Dawson D. Daytime melatonin and temazepam in young adult humans: equivalent effects on sleep latency and body temperature. J Physiol. 1999;514:905-914.

18. Kräuchi K, Cajochen C, Pache M, Flammer J, Wirz-Justice A. Thermoregulatory effects of melatonin in relation to sleepiness. Chronobiol Int. 2006;23:475-484.

19. Kräuchi K, Cajochen C, Wirz-Justice A. A relationship between heat loss and sleepiness: effects of postural change and melatonin administration. J Apply Physiol. 1997;83:134-139.

20. Reid C, van den Heuvel C, Dawson D. Day-time melatonin administration: effects on core temperature and sleep onset latency. J Sleep Res. 1996;5:150-154.

21. Strassman RJ, Qualls CR, Lisansky EJ, Peake GT. Elevated rectal temperature produced by all-night bright light is reversed by melatonin infusion in men. J Apply Physiol. 1991;71:2178-2182.

22. Zhdanova IV, Wurtman RJ. Efficacy of melatonin as a sleep-promoting agent. J Biol Rhythms. 1997;12:644-650.

23. Achterberg J, McGraw P, Lawlis GF. Rheumatoid arthritis: a study of relaxation and temperature biofeedback training as an adjunctive therapy. Biofeedback Self-Regul. 1981;6:207-223.

24. Ancoli-Israel S, Seifert AR, Lemon M. Thermal biofeedback and periodic movement in sleep: patients' subjective reports and a case study. Biofeedback Self-Regul. 1986;11:177-188.

25. Benjamin J, Schofield W. Treatment of sleep onset insomnia with finger temperature feedback and self-monitoring: a case study. Proceedings of the Biofeedback Society of America, Ninth Annual Meeting, Denver, CO. Applied Psychophysiol Biofeedback. 1978;3:59.

26. Harden RN, Houle TT, Green S, et al. Biofeedback in the treatment of phantom limb pain: a time-series analysis. Applied Physiopathol Biofeedback. 2005;30:83-93.

27. Karavidas MK, Tsai Pei-Shan, Yucha C, McGrady A, Lehrer PM. Thermal biofeedback for primary Raynaud's phenomenon: a review of the literature. Applied Psychophysiol Biofeedback. 2006;31:203-216.
28. Linden W, Moseley JV. The efficacy of behavioural treatments for hypertension. Applied Psychophysiol Biofeedback. 2006;31:51-63.

29. Nestoriuc Y, Martin A. Efficacy of biofeedback for migraine: a metaanalysis. Pain. 2007;128:111-127.

30. Viens M, Swingle P, De Koninck J. The treatment of periodic leg movements using thermal biofeedback: failure to replicate. J Sleep Res. 1989;18:318

31. De Koninck J, Grenier J, Castonguay M, Forest G, Gosselin A, Swingle PG. Self-regulation of body temperature and sleep latency. J Sleep Res. 2002;11:49.

32. De Koninck J, Swingle PG, Hébert M, Couture-Côté C, Côté Y. Selfregulation of core body temperature and sleep. J Sleep Res. 1993; 22:399.

33. Forest G, Grenier J, Couture C, Côté Y, Swingle P, De Koninck J. La température corporelle et l'endormissement. Neuropsychol Clin. 2004;33:41.

34. Forest G, Lamarche L, Grenier J, Castonguay M, De Koninck J. Thermal biofeedback and sleep: limited effects and methodological challenges. Sleep. 2004;27:A45.

35. Lamarche L, Forest G, Forget D, Gauthier A-K, De Koninck J. Presleep hand temperature biofeedback and sleep architecture. Paper presented at the World Congress Chronobiology, Sapporo, Japan, Sep 2003.

36. Freedman RR. Physiological mechanisms of temperature biofeedback. Biofeedback Self-Regul. 1991;16:95-115.

37. Johnson JM, Rowell LB, Brengelmann G. Modification of the skin blood flow-body temperature relationship by upright exercise. J Applied Physiol. 1974;37(6):880-886.

38. Rechtschaffen A, Kales A. A manual of standardized terminology, techniques and scoring system for sleep stages of human subjects. Los Angeles: Brain Information/Brain Research Institute, UCLA; 1968.

39. van den Heuvel CJ, Ferguson S, Dawson D. Attenuated thermoregulatory response to mild thermal challenge in subjects with sleep-onset insomnia. Sleep. 2006;29:1174-1180.

40. Lushington K, Dawson D, Lack L. Core body temperature is elevated during constant wakefulness in elderly poor sleepers. Sleep. 2000;23: 504-510.
ChronoPhysiology and Therapy

\section{Publish your work in this journal}

ChronoPhysiology and Therapy is an international, peer-reviewed, open access journal focusing on research into the cyclic variations and rhythmicity in physiological processes in the body and the research and development and optimal timing of administration of therapeutic targets to achieve improved outcomes and quality of life for the patient. The

\section{Dovepress}

manuscript management system is completely online and includes a very quick and fair peer-review system. Visit http://www.dovepress.com/ testimonials.php to read real quotes from published authors. 